\title{
¿Cómo cambió la enseñanza-aprendizaje de las asignaturas prácticas en el área de tecnologías de la información con la \\ covid-19?
}

How Did the Teaching and Learning of Practical Courses in Information Technology Change with COVID-19?

Como o ensino-aprendizagem de disciplinas práticas da área de tecnologia da informação mudou com a covid-19?

José Balderas Solís

Universidad Autónoma de Tamaulipas, México

josebalderas97@gmail.com https://orcid.org/0000-0001-6293-7825

Ramón Ventura Roque Hernández Universidad Autónoma de Tamaulipas, México rvHernandez@uat.edu.mx https://orcid.org/0000-0001-9727-2608

Adán López Mendoza

Universidad Autónoma de Tamaulipas, México

aLopez@uat.edu.mx https://orcid.org/0000-0003-4801-640X

Rolando Salazar Hernández

Universidad Autónoma de Tamaulipas, México rsalazar@docentes.uat.edu.mx https://orcid.org/0000-0001-5879-4083

Carlos Manuel Juárez Ibarra Universidad Autónoma de Tamaulipas, México cJuarez@docentes.uat.edu.mx https://orcid.org/0000-0003-4310-8938 

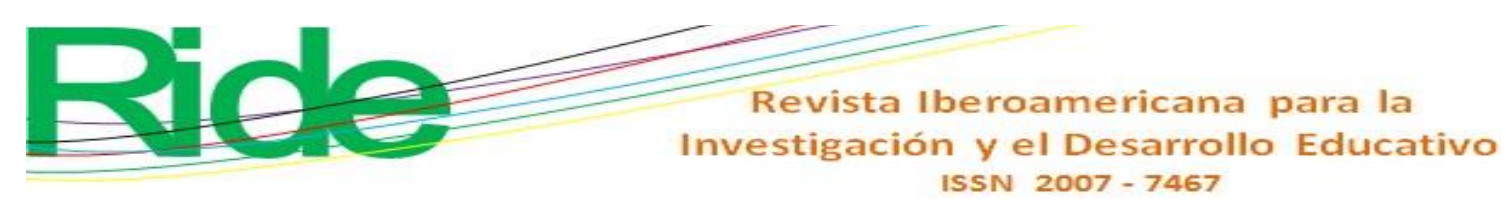

\section{Resumen}

Introducción: Durante la contingencia sanitaria por covid-19, la educación migró hacia ambientes virtuales que permitieron el aislamiento social necesario para evitar la propagación del virus. Para todos hubo un periodo de adaptación a la nueva modalidad. Sin embargo, los docentes y alumnos con asignaturas de laboratorio enfrentaron un reto todavía mayor: el de abordar contenidos prácticos desde la virtualidad, es decir, sin estar físicamente presentes en un laboratorio. Objetivo: El presente artículo se sitúa en este contexto con el objetivo de lograr un acercamiento a la caracterización de la enseñanza y aprendizaje de las asignaturas prácticas universitarias en el área de tecnologías en los tiempos de covid-19. Método: Se presenta el estudio de caso de una universidad pública mexicana (que por confidencialidad se denominará UNX) en la carrera de licenciatura en Tecnologías de la Información, en donde el trabajo de laboratorio de hardware y software está implícito en la mayoría de las asignaturas. En esta investigación se realizaron entrevistas en profundidad a cinco profesores y se aplicaron cuestionarios con preguntas abiertas a seis alumnos universitarios del área de tecnologías de la información. Durante el semestre de primavera 2020, los cinco profesores impartieron materias cuyos contenidos implicaban prácticas de laboratorio. Los seis alumnos cursaron esas mismas materias con estos docentes. Ni alumnos ni profesores conocían que la investigación abarcaría ambas perspectivas. En las entrevistas con los docentes se abordaron los siguientes aspectos: contenidos, labor docente, alumnos, dinámica de la clase y modalidad en línea. Los cuestionarios con los alumnos solamente se abordaron los últimos tres aspectos. Para el procesamiento de los datos se utilizó el software MAXQDA versión 20. Resultados: A pesar de que la mayor parte de los contenidos se pudo cubrir con éxito, hubo temas prácticos que pudieron profundizarse más, y otros que no se abordaron. La comunicación entre profesores y alumnos fue fluida y explícita. Los docentes encontraron muy conveniente la modalidad en línea y coincidieron en que preferirían impartir sus clases de esta manera aun en escenarios futuros, libres de pandemia. En este sentido, aceptaron que su experiencia docente en línea podría mejorar, para lo cual deberían incrementar su planeación y diversificar los formatos de sus sesiones y materiales de clase. Por su parte, los estudiantes coincidieron en que la forma de evaluar y la comunicación con los docentes fueron buenas. Sin embargo, reconocieron áreas de oportunidad y concluyeron que no les gustaría tomar sus clases completamente en línea cuando termine la contingencia por covid-19. Conclusiones: La docencia universitaria de las asignaturas prácticas en el área de tecnologías en los tiempos 


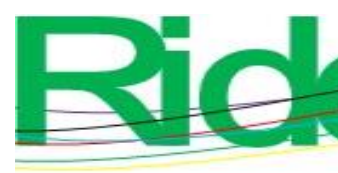

Revista Iberoamericana para la
Investigación y el Desarrollo Educativo
ISSN $2007-7467$

del covid-19 se realizó de manera virtual en un ambiente de buena comunicación entre docentes y alumnos. Los contenidos, materiales didácticos y forma de impartirlos tuvieron que adaptarse. Tanto alumnos como docentes reconocen que fue una buena experiencia, pero que es susceptible de mejorar todavía más a través del compromiso personal y colectivo de todos los actores involucrados.

Palabras clave: aprendizaje en línea, asignaturas de enseñanza profesional, enseñanza superior, epidemia.

\section{Abstract}

Introduction: During the health contingency due to COVID-19, education migrated to virtual environments that allowed the necessary social isolation to prevent the spread of the virus. There was a period of adaptation to the new modality for everyone. However, professors and students with lab courses faced an even greater challenge: that of addressing practical content from virtual scenarios, that is, without being physically present in a laboratory. Objective: This paper is developed in this context with the objective of achieving an approach to the characterization of the teaching and learning of university practical courses in technologies in the times of COVID-19. Method: A case study of a Public Mexican University (named UNX, for confidentiality purposes) and its bachelor's degree in Information Technology, where the hardware and software laboratory work is implicit in most courses is presented. In this research, in-depth interviews were conducted with five professors, and questionnaires with open questions were applied to six university students in Information Technology. During Spring 2020, the five professors taught subjects whose contents involved laboratory practices. The six students studied the same courses with these professors. Neither students nor professors knew that the research would encompass both perspectives. In the interviews with the professors, the following aspects were addressed: course content, teaching work, students, class dynamics and 'online' mode. In the questionnaires with the students, only the last three aspects were addressed. For data processing, MAXQDA version 20 software was used. Results: Even though most of the content was covered successfully, there were practical topics that could be deepened further and others that were not addressed. Communication between professors and students was fluid and explicit. The professors found the online mode very convenient and agreed that they would prefer to teach their classes in this way even in future scenarios, free of 


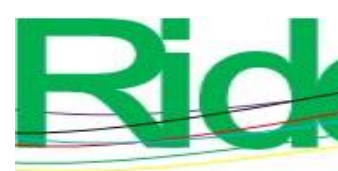

Revista Iberoamericana para la Investigación y el Desarrollo Educativo ISSN $2007-7467$

pandemics. They accepted that their online teaching experience could improve, for which they should increase their planning and diversify the formats of their sessions and class materials. Students agreed that the evaluations and communication with the professors were good. However, they identified some areas of opportunity and concluded that they would not like to take their classes completely online when the COVID19 contingency ends. Conclusions: The university teaching of practical courses in technologies in the times of COVID-19 was carried out virtually in an environment of good communication between professors and students. The contents, learning materials, and the way of teaching had to be adapted. Both students and professors recognize that it was a good experience, but that it is susceptible to further improvement through the personal and collective commitment of all the actors involved.

Keywords: electronic learning, vocational training subjects, higher education, epidemics.

\section{Resumo}

Introdução: Durante a contingência de saúde devido ao covid-19, a educação migrou para ambientes virtuais que permitiam o isolamento social necessário para prevenir a propagação do vírus. Para todos houve um período de adaptação à nova modalidade. Porém, professores e alunos com disciplinas de laboratório enfrentaram um desafio ainda maior: o de abordar conteúdos práticos a partir da virtualidade, ou seja, sem estar fisicamente presente em um laboratório. Objetivo: Este artigo se situa neste contexto com o objetivo de abordar a caracterização do ensino e da aprendizagem de disciplinas universitárias práticas na área de tecnologias na época de covid-19. Método: O estudo de caso de uma universidade pública mexicana (que se chamará UNX devido ao sigilo) é apresentado no curso de Bacharelado em Tecnologia da Informação, onde o trabalho do laboratório de hardware e software está implícito na maioria das disciplinas . Nesta pesquisa, foram realizadas entrevistas em profundidade com cinco docentes e aplicados questionários com questões abertas a seis universitários da área de tecnologia da informação. Durante o semestre da primavera de 2020, os cinco professores ministraram disciplinas cujos conteúdos envolviam práticas laboratoriais. Os seis alunos estudaram as mesmas matérias com esses professores. Nem os alunos nem os professores sabiam que a pesquisa abarcaria ambas as perspectivas. Nas entrevistas com os professores foram abordados os seguintes aspectos: conteúdo, trabalho docente, alunos, dinâmica das aulas e modalidade online. Os questionários com os alunos 


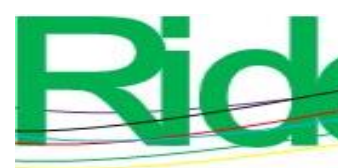

Revista Iberoamericana para la
Investigación y el Desarrollo Educativo
ISSN $2007-7467$

abordaram apenas os três últimos aspectos. Para o processamento dos dados, foi utilizado o software MAXQDA versão 20. Resultados: Apesar de a maior parte dos conteúdos ter sido abordada com sucesso, houve tópicos práticos que puderam ser explorados mais a fundo e outros que não foram abordados. A comunicação entre professores e alunos era fluida e explícita. Os professores acharam a modalidade online muito conveniente e concordaram que prefeririam ministrar suas aulas dessa forma mesmo em cenários futuros, livres de pandemias. Nesse sentido, aceitaram que sua experiência de ensino online pudesse melhorar, para o que deveriam aumentar seu planejamento e diversificar os formatos de suas sessões e materiais didáticos. Por sua vez, os alunos concordaram que a forma de avaliação e comunicação com os professores foi boa. No entanto, eles reconheceram áreas de oportunidade e concluíram que não gostariam de ter suas aulas totalmente online quando a contingência covid-19 terminar. Conclusões: O ensino universitário de disciplinas práticas na área de tecnologias na época de covid-19 era realizado virtualmente em ambiente de boa comunicação entre professores e alunos. Os conteúdos, materiais didáticos e a forma de transmissão tiveram que ser adaptados. Alunos e professores reconhecem que foi uma boa experiência, mas que pode ser aprimorada por meio do comprometimento pessoal e coletivo de todos os atores envolvidos.

Palavras-chave: aprendizagem online, disciplinas de educação profissional, ensino superior, epidemia.

Fecha Recepción: Agosto 2020

Fecha Aceptación: Enero 2021

\section{Introducción}

La covid-19 llegó a México en medio de un periodo escolar que parecía normal en su transcurso. Sin embargo, la consecuente contingencia sanitaria decretada por las autoridades el 14 de marzo de 2020 obligó a las instituciones educativas a suspender actividades presenciales, lo que ocasionó que tuvieran poco tiempo para prepararse y reaccionar. Los directivos y administradores tuvieron que realizar adaptaciones de emergencia en los calendarios escolares y en las estrategias para continuar con la vida académica. Igualmente, los docentes tuvieron que adaptar su cátedra presencial a medios virtuales con variados niveles de dificultad y se enfrentaron a los retos y al estrés que supone el trabajo desde casa en medio de una pandemia. Los alumnos, por su parte, no estuvieron exentos de enfrentar los desafíos de estudiar desde su casa. 


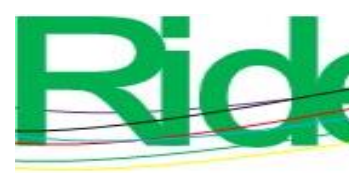

Revista Iberoamericana para la Investigación y el Desarrollo Educativo ISSN 2007 - 7467

En este escenario de cambios, ajustes repentinos y nuevas formas de trabajo, el internet se erigió como el elemento central de comunicación que hizo posible que la docencia no quedara pausada indefinidamente. Las clases en línea se adoptaron como la alternativa más adecuada para dar continuidad a las actividades educativas. Fue así como diversas plataformas y modalidades comenzaron a implementarse en un plan tecnológico de emergencia que implicó serios desafíos para instituciones, docentes y alumnos de todos los niveles educativos. En el caso concreto de una universidad pública mexicana (que por confidencialidad se denominará UNX), hubo libertad para utilizar cualquier plataforma o medio digital en cada una de sus sedes. Concretamente, en la Facultad X se adoptó Microsoft Teams como herramienta oficial de aprendizaje y comunicación. Por indicaciones explícitas de la dirección, las sesiones regulares de clase deberían impartirse en línea y en vivo en los horarios oficiales que habían sido asignados al inicio del semestre, cuando todavía no se vislumbraba la posible aparición de una pandemia. Esto significaba que alumnos y maestros seguirían reuniéndose los días y horas acostumbrados, pero ahora virtualmente a través de Microsoft Teams.

Aunque Office 365 y sus herramientas habían estado disponibles para alumnos y maestros gratuitamente desde hacía algún tiempo, pocos de ellos habían utilizado Microsoft Teams. Apenas en febrero de 2020 había iniciado un diplomado en línea para docentes de varias universidades en el cual se promovía su uso. Varios maestros de la UNX lo estaban cursando cuando inició la contingencia. Sin embargo, sus destrezas se mantenían en niveles principiantes. Los grupos de alumnos que estos maestros atendían en la universidad también habían tenido algún contacto con Microsoft Teams, ya que los maestros habían comenzado a poner en práctica lo aprendido para cumplir con las tareas que el diplomado les requería.

Al inicio de la contingencia, las clases se suspendieron durante una semana en la UNX con el objetivo de impartir talleres presenciales intensivos de Microsoft Teams dirigidos a todos los docentes de la facultad. En estas jornadas de capacitación se abordaron las temáticas básicas de creación de equipos, uso del chat, inicio, fin y grabación de sesiones en vivo, así como programación de las sesiones en el calendario y compartición de archivos. Fue así como la semana siguiente todas las clases se reanudaron en línea a través de Microsoft Teams.

Para docentes y alumnos hubo un periodo de adaptación y una curva de aprendizaje a la nueva modalidad. No obstante, quienes impartieron o cursaron asignaturas prácticas tuvieron un reto todavía mayor: el de abordar contenidos prácticos sin estar físicamente presentes en un laboratorio. Quizá el caso más representativo de este escenario en la UNX se 


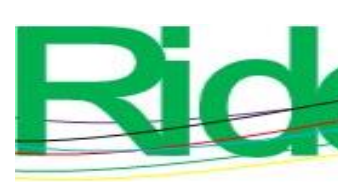

Revista Iberoamericana para la
Investigación y el Desarrollo Educativo
ISSN $2007-7467$

encuentra en la licenciatura en Tecnologías de la Información, pues el trabajo de laboratorio está implícito en la mayoría de las asignaturas de este programa educativo.

El presente artículo, por tanto, se sitúa justamente en este contexto con el objetivo de lograr un acercamiento a la caracterización de la docencia universitaria de las asignaturas prácticas en el área de tecnologías en los tiempos de covid-19.

Microsoft Teams es una plataforma tecnológica incluida en Office 365 en la cual se pueden crear equipos de clase virtuales y canales de discusión. También es posible asignar y revisar tareas, aplicar exámenes, compartir recursos didácticos como documentos en diversos formatos, videos, tutoriales y presentaciones. Una de las principales ventajas de esta plataforma es que permite a alumnos y docentes interactuar de manera virtual en tiempo real. Además, las sesiones pueden ser grabadas en la aplicación Stream para ser consultadas posteriormente. Esta plataforma ofrece funcionalidades que son de gran ayuda para el ambiente académico, pues favorece la ubicuidad en el proceso de enseñanza-aprendizaje. Teams funciona en computadoras de escritorio y portátiles, así como en celulares y tabletas con sistemas operativos Android o iOs.

Se ha encontrado que los estudiantes han adoptado Microsoft Teams rápidamente a pesar de las preocupaciones de que ocurriera lo contrario (Hodgson y Hagan, 2020). También existe referencia en la literatura de que Microsoft Teams se ha implementado para revitalizar el entrenamiento de los estudiantes que trabajan. Esto es porque se ha encontrado que, al utilizarlo, los estudiantes son más productivos e informados y que sus vidas se han facilitado al contar con las herramientas que requieren (Belvin, 2018). Teams también se ha utilizado para compartir recursos educativos en línea (Halstead-Nussloch, Dickson, Greer, Siddiqui y Tumuluri, 2019) y para llevar la experiencia presencial del campus a las clases en línea (Owens, Weismann y Suskin, 2019). Por otra parte, durante la pandemia, Microsoft Teams ha sido utilizado con intenciones educativas (Pather et al., 2020; Sinha, Sharma y Cullen, 2020; Tofade y Daftary, 2020) y de telemedicina (Ghosh, Gupta y Misra, 2020; Price et al., 2020).

De acuerdo con el trabajo de Haleem, Javaid, Vaishya y Deshmukh (2020), la covid-19 está dando una nueva importancia a varias áreas de investigación académica. Algunas de ellas son los fenómenos sociales, el surgimiento de un nuevo lugar de trabajo y de una nueva cultura laboral, así como la revolución de las tecnologías de información. En esta última área, Haleem et al. (2020) subrayan las nuevas formas de aprendizaje, las clases en línea y el uso de laboratorios virtuales a través de vídeo y otros materiales de apoyo. 


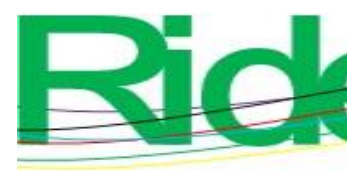

Revista Iberoamericana para la
Investigación y el Desarrollo Educativo
ISSN $2007-7467$

La educación médica se ha identificado como un área eminentemente práctica y presencial que ha sido afectada por la contingencia sanitaria de covid-19 (Ferrario et al., 2020; Panesar et al., 2020). En este sentido, Ferrario et al. (2020) brindan cuatro recomendaciones que, al generalizarlas, bien podrían ser útiles para cualquier otra área de conocimiento práctico: 1) la seguridad es primero, por lo que se debe reducir la presencia del personal no esencial, así como el contacto físico, 2) fortalecer el uso de las tecnologías a través de clases en línea, grabaciones, videoconferencias, clubes semanales extracurriculares y simuladores, 3) motivar a los estudiantes para que utilicen su tiempo libre en leer, estudiar y aumentar su producción académica con el apoyo diario de sus tutores o profesores, y 4) proveer apoyo psicológico a los estudiantes.

Ping, Fudong y Zheng (2020) destacan algunos problemas que surgen en la modalidad en línea como sustituto de la educación presencial en tiempos de covid-19: es difícil para los docentes saber si los estudiantes están poniendo atención o están holgazaneando. Es probable que algunos alumnos no puedan realizar preguntas si no entienden algo, y además los estudiantes requieren autodisciplina, lo que puede ser difícil de lograr para ellos. De esta manera, Ping, Fudong y Zheng (2020) proponen el aprendizaje basado en problemas como un método efectivo para la enseñanza en línea, especialmente en el nivel universitario y en el área de tecnologías. En este enfoque hay tres etapas: aprendizaje autónomo por parte del estudiante, encuentros virtuales en vivo y ejercicios en línea. La idea central es enseñar utilizando problemas y preguntas. En este proceso, el profesor puede guiar a los estudiantes, resolver conjuntamente los problemas con ellos, o bien dejar que ellos los resuelvan por sí mismos.

Para Feng, Yu, Hu y Fan (2020), los docentes, además de enfrentar los retos de conocer cómo utilizar el nuevo software para impartir sus clases, tienen que convertir sus materiales de aprendizaje en versiones electrónicas y ajustar el ritmo de la clase. Todo esto sin contar con el estrés que puede causarles la falta de respuesta de los estudiantes al interactuar con ellos. Por otra parte, señalan que, como la enseñanza en línea es completamente diferente a la enseñanza presencial, las universidades deben establecer estrategias para apoyar este proceso. Por ejemplo, es recomendable contar con un equipo de supervisión, apoyo, calidad y control de las clases en línea. Asimismo, es importante elegir una plataforma eficiente y con las funcionalidades necesarias para proveer la educación virtual. También los docentes deben capacitarse para aprovechar al máximo la plataforma utilizada, diseñar planes de aprendizaje y adoptar métodos diversificados y flexibles para 


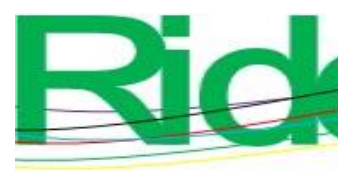

Revista Iberoamericana para la
Investigación y el Desarrollo Educativo
ISSN $2007-7467$

organizar la enseñanza. Finalmente, estos autores señalan que es importante analizar los resultados de la experiencia y realizar las evaluaciones y acciones derivadas que resulten pertinentes.

Por su parte, después de analizar los efectos de la covid-19 en las actividades cotidianas, Cerf (2020) menciona que el internet y la Web, las videoconferencias, las herramientas de colaboración, el correo electrónico y las redes sociales son los medios principales para los negocios, la interacción social y el entretenimiento durante la pandemia. Posteriormente, se cuestiona si en la sociedad poscovid-19, el trabajo y las actividades académicas a distancia se volverán más comunes e incluso preferidas por las personas en lugar de las presenciales.

Es claro que la aparición de la covid-19 ha modificado gran parte de la actividad humana y ha dado paso a nuevas interacciones que es necesario estudiar. También es cierto que hay muchas interrogantes que han surgido y quedan pendientes por contestar. Concretamente, en el ámbito educativo resulta relevante conocer cómo se han llevado a cabo los procesos de enseñanza-aprendizaje durante la pandemia. De esta manera, será posible comprenderlos mejor y preparar escenarios futuros en donde se puedan fortalecer las áreas de oportunidad que se detecten.

Este artículo, por ende, presenta una investigación realizada con el objetivo de lograr un acercamiento a la caracterización de la enseñanza y aprendizaje de las asignaturas prácticas universitarias en el área de tecnologías de la información en los tiempos de covid19. El resto de este documento está organizado de la siguiente manera: a continuación, se presenta el método que se siguió, posteriormente se muestran los resultados y su discusión, y finalmente se abordan las conclusiones y trabajos futuros.

\section{Método}

\section{Tipo de estudio}

Se utilizó un enfoque cualitativo en donde se realizaron entrevistas en profundidad a cinco profesores universitarios del área de tecnologías de la información. Durante el semestre de primavera 2020, los cinco impartieron materias cuyos contenidos implicaban prácticas de laboratorio. También participaron seis alumnos de esta misma área que cursaron estas mismas materias con los docentes entrevistados. 

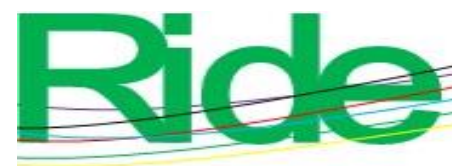

Revista Iberoamericana para la Investigación y el Desarrollo Educativo

ISSN $2007-7467$

Primero, cada uno de los profesores participantes fue contactado para agendar una cita individual y virtual a través de la plataforma Microsoft Teams. Llegado el momento del encuentro, a cada uno se le explicó el propósito de la entrevista y se le solicitó su autorización para ser parte de este estudio. Las cinco entrevistas con los docentes fueron grabadas y produjeron material en video de 198.20 minutos en total. Los seis alumnos participantes fueron contactados a través de Whatsapp y/o a través de Microsoft Teams, y se les explicó en qué consistiría su participación. Una vez que aceptaron, se les proporcionó un enlace con un cuestionario que contenía preguntas abiertas, las cuales contestaron a través de la plataforma de Google Forms. En algunos casos se contactó nuevamente a los estudiantes por Whatsapp o por Teams para clarificar o ampliar algunas respuestas.

\section{Perfil de los entrevistados y de las asignaturas prácticas en las que participaron durante la contingencia sanitaria}

Se contó con la participación de cinco docentes, los cuales fueron identificados con los códigos D1 a D5. En la tabla 1 se muestran las asignaturas prácticas y los contenidos que cada uno impartió durante el semestre de primavera de 2020. 


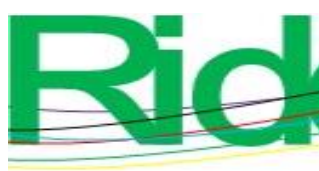

Revista Iberoamericana para la Investigación y el Desarrollo Educativo ISSN 2007 - 7467

Tabla 1. Perfil de los profesores participantes y de las asignaturas prácticas que impartieron

\begin{tabular}{|c|c|c|c|}
\hline $\begin{array}{l}\text { Docentes } \\
\text { entrevistados }\end{array}$ & $\begin{array}{lr}\text { Antigüedad } & \text { (en } \\
\text { años) } & \text { como } \\
\text { docente en la } \\
\text { UNX }\end{array}$ & $\begin{array}{l}\text { Asignaturas con contenidos } \\
\text { prácticos de laboratorio } \\
\text { impartidas en el semestre de } \\
\text { primavera } 2020\end{array}$ & $\begin{array}{l}\text { Contenidos prácticos de } \\
\text { laboratorio que aborda } \\
\text { la asignatura }\end{array}$ \\
\hline \multirow[t]{4}{*}{ D1 } & \multirow[t]{4}{*}{19} & $\begin{array}{l}\text { Fundamentos de la } \\
\text { informática y metodología } \\
\text { de la programación }\end{array}$ & $\begin{array}{l}\text { Programación por lotes } \\
\text { (Batch) }\end{array}$ \\
\hline & & $\begin{array}{l}\text { Seguridad e integridad de } \\
\text { redes }\end{array}$ & 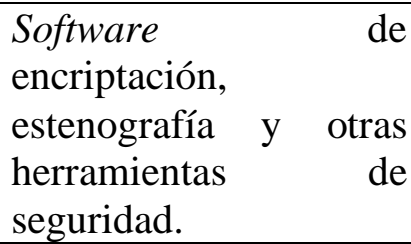 \\
\hline & & Sistemas operativos cliente & $\begin{array}{l}\text { Virtual Box para } \\
\text { instalar los sistemas } \\
\text { operativos }\end{array}$ \\
\hline & & $\begin{array}{ll}\text { Implantación } & \text { y } \\
\text { configuración de redes }\end{array}$ & $\begin{array}{l}\text { Práctica con routers, } \\
\text { antenas y Cisco Packet } \\
\text { Tracer }\end{array}$ \\
\hline D2 & 19 & Diseño de base de datos & Microsoft Access \\
\hline D3 & 1 & Programación intermedia & C\# \\
\hline \multirow[t]{2}{*}{ D4 } & \multirow[t]{2}{*}{1} & $\begin{array}{l}\text { Programación intermedia- } \\
\text { avanzada }\end{array}$ & Angular \\
\hline & & $\begin{array}{l}\text { Desarrollo de bases de datos } \\
\text { avanzado }\end{array}$ & Benchmark \\
\hline D5 & 24 & $\begin{array}{l}\text { Mantenimiento de equipo de } \\
\text { cómputo }\end{array}$ & $\begin{array}{l}\text { Prácticas de ensamble, } \\
\text { diagnóstico } \\
\text { mantenimiento } \\
\text { hardware }\end{array}$ \\
\hline
\end{tabular}

Fuente: Elaboración propia

También se contó con la participación de seis estudiantes, los cuales cursaron exactamente las mismas materias que impartieron los docentes entrevistados. Los alumnos fueron identificados con los códigos E1 a E6. En la tabla 2 se muestran sus perfiles. 


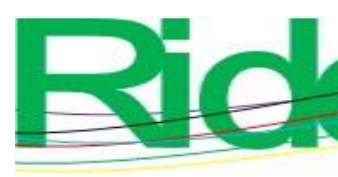

Revista Iberoamericana para la Investigación y el Desarrollo Educativo ISSN $2007-7467$

La duración en minutos de las entrevistas con cada profesor fue la siguiente: D1 (40.03), D2 (47.29), D3 (35.58), D4 (31.25) y D5 (44.05).

\section{Análisis de datos}

Las entrevistas con los docentes fueron introducidas y transcritas en el software MaxQDA 20, en donde se realizaron las codificaciones correspondientes y se extrajeron categorías a través de las cuales se presentan los resultados. Las respuestas de los estudiantes a las preguntas abiertas también se introdujeron en MaxQDA 20 en documentos de Microsoft Word. En el software se realizó una tabla de resumen para recabar los puntos centrales. Este resumen fue exportado a Excel y se utilizó para estructurar las respuestas obtenidas.

\section{Resultados}

\section{Contenidos}

Los docentes utilizaron presentaciones en Power Point (D1, D2, D4), libros electrónicos (D2, D3, D5), videos (D2, D4, D5) y sitios de internet (D3) como material didáctico durante la contingencia. Aunque en algunas asignaturas el tiempo alcanzó perfectamente para cumplir los objetivos — bases de datos (D5), diseño de bases de datos (D2), seguridad e integridad de redes (D1), implementación de redes (D1)—, en otras asignaturas fue necesario realizar ajustes en función de cada asignatura (tabla 4).

Tabla 4. Asignaturas y ajustes realizados en sus contenidos durante la contingencia sanitaria

\begin{tabular}{|l|l|}
\hline \multicolumn{1}{|c|}{ Asignatura } & \multicolumn{1}{c|}{ Ajustes } \\
\hline $\begin{array}{l}\text { Fundamentos de la informática y } \\
\text { metodología de la programación }\end{array}$ & $\begin{array}{l}\text { Se comenzaron los temas, pero faltó profundizar } \\
\text { más. }\end{array}$ \\
\hline Sistemas operativos cliente & $\begin{array}{l}\text { Faltó práctica de Linux. Se cubrió el tema } \\
\text { teóricamente. }\end{array}$ \\
\hline Programación intermedia & $\begin{array}{l}\text { Faltó práctica en el acceso a base de datos con C\#. } \\
\text { Se cubrió el tema superficialmente. }\end{array}$ \\
\hline Programación intermedia avanzada & $\begin{array}{l}\text { Faltó práctica en el tema de conexiones a bases de } \\
\text { datos. }\end{array}$ \\
\hline $\begin{array}{l}\text { Mantenimiento de equipo de } \\
\text { cómputo }\end{array}$ & $\begin{array}{l}\text { Faltó hacer prácticas de laboratorio, instalación y } \\
\text { mantenimiento. No se abordó el tema de } \\
\text { dispositivos de comunicación. }\end{array}$ \\
\hline
\end{tabular}

Fuente: Elaboración propia 


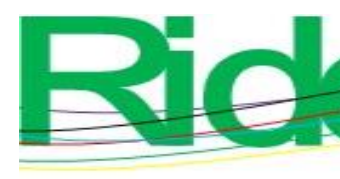

Revista Iberoamericana para la
Investigación y el Desarrollo Educativo
ISSN $2007-7467$

Al respecto, D4 comentó que “quité un examen y lo unifiqué con otro (...). También usé la semana previa al inicio de clases en línea para que los alumnos instalaran el software que íbamos a usar". D2 expresó: "Yo pensaba que a lo mejor no nos iba a alcanzar el tiempo. Pero (por) el hecho de tener las clases en línea (...) no se nos vieron interrumpidos los días de clase. Presencialmente siempre hay algún evento o actividad que los alumnos tienen que atender y eso retrasa el programa. En esta ocasión, al contrario, tuvimos la mayoría de las sesiones. No hubo interrupción en los días de clase (...). Tuvimos la oportunidad de hacer más ejercicios".

\section{Profesores y su labor docente}

\section{¿Cómo era el entorno de los docentes que impartían materias prácticas?}

Todos los docentes entrevistados contaban con una computadora y un celular mientras trabajaban desde casa. Aunque Microsoft Teams puede ejecutarse en ambos dispositivos, los profesores siempre utilizaron su computadora para unirse a sus sesiones virtuales. El celular lo usaron principalmente para responder los mensajes de chat que los alumnos les hacían llegar después de la sesión de clase. Solamente uno de los docentes (D1) usó el celular para ingresar a su sesión debido a un problema de conexión. Sin embargo, lo hizo solamente para encargar una tarea, por lo que se desconectó rápidamente. Los cinco docentes entrevistados contaban con internet en casa con las siguientes velocidades: 1, 10, 20, 25 y $40 \mathrm{Mb}$, y ninguno reportó problemas graves con su conexión. Solamente dijeron haber tenido problemas técnicos relacionados con el internet o con el acceso a la plataforma en máximo dos ocasiones. Para cumplir con su docencia, ningún profesor enfrentó retos serios debido a su ámbito familiar. Sin embargo, dos de ellos tuvieron que organizar sus tiempos y espacios con sus hijos para llevar a cabo su docencia (D2, D5). Esto fue porque los hijos de estos maestros también cursaban clases en línea a la misma hora que ellos impartían su clase desde casa. Los otros tres profesores contaban ya con un espacio adaptado para trabajar.

\section{¿A qué retos se enfrentaron los profesores y cómo los solucionaron?}

Uno de los principales retos docentes estaba relacionado con los alumnos. Sobre esto, D4 comentó: 


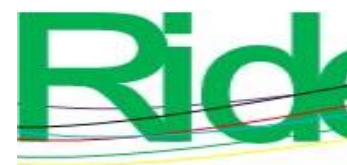

Revista Iberoamericana para la Investigación y el Desarrollo Educativo ISSN $2007-7467$

Al principio tuve incertidumbre. Me preocupé porque a través de una pequeña encuesta les pregunté a mis alumnos qué modalidad preferían y casi el $100 \%$ me contestó "presencial". Platicando con ellos, me decían que los profesores les pedían más trabajo en línea, que no los dejaban descansar. Me preocupaba que se quedaran con esa imagen de las clases en línea. Yo les decía: "Esto es un proceso de contingencia y hay muchas áreas de oportunidad". Los profesores no nos coordinábamos y hubo cosas que no se hablaron. Para mí fue un reto no tener la visibilidad de cómo estábamos trabajando todos. Me preocupaba la reacción de los chavos (...). Otro reto fue el responder las dudas a través del chat. Hubo un momento en que me escribían a todas horas.

D3 también se preocupaba por los estudiantes:

El primer reto fue captar la atención de los muchachos. Cuando estoy en el salón (físicamente) estoy viendo quién al menos te entrega una mirada, quién está abajo. ¿En línea cómo voy a entender que ellos están absorbiendo lo que estoy comentando? Fue algo muy complicado el soltar esa responsabilidad, pues es una aventura. Otro reto fue encontrar la forma de que la empatía no se volviera cómplice de la irresponsabilidad. Tuve una experiencia con un alumno que me dijo: "Profe, me voy a ir a almorzar, pero ahorita regreso". Otro alumno me dijo: “(En mi empleo) me cambiaron el horario por la contingencia. Entro a las siete y salgo a las tres y no puedo conectarme a la clase","

D2 también comentó:

En algunas ocasiones no me podía percatar si el alumno estaba entendiendo lo que yo le quería decir. Aunque se puede compartir la pantalla y casi llevarlo de la mano, hay algunos distractores que los muchachos tienen y no logran captar (...). Al momento de dar las clases y de estar conectado con los muchachos, al principio sí había un poquito de que lo querían tomar a juego.

D2 también refirió los problemas técnicos y de tiempo:

Otra situación fue la dificultad al momento de evaluar o revisar los exámenes. Me lo enviaban en una versión que yo no podía abrir. La plataforma se tardaba demasiado para cargar los exámenes y los trabajos de los alumnos. Era muy recurrente el hecho que los muchachos se quejaran de que no lo podían enviar 


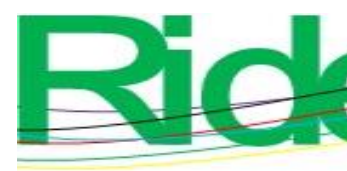

Revista Iberoamericana para la
Investigación y el Desarrollo Educativo
ISSN $2007-7467$

por ahí. También los tiempos. Para el examen práctico hay cierto tiempo y con estas situaciones no te podías ajustar a ese tiempo.

Para D1, el principal reto fue la preparación de los temas:

Fue un reto ajustar los contenidos. Los temas nuevos no los tenía preparados para darlos de forma virtual (...). De forma presencial les explicaba un tema y lo poníamos en práctica (...). En línea tuve que crear contenidos nuevos y otros adecuarlos, (...) ya que no los iba a estar acompañando (a los alumnos).

Para D5, su principal reto fue la realización de prácticas de hardware: "Prácticas (de mantenimiento de equipo) no pudimos hacer gran cosa. Nos direccionamos a preguntas, observaciones y recordatorios. No podíamos hacer nada práctico”.

Los docentes hicieron frente a estos retos con su creatividad y el uso de la herramienta Microsoft Teams, a través de la cual podían interactuar con los alumnos ya sea en las sesiones virtuales o a través del chat. D4 tuvo "que poner un horario para la atención a alumnos". Una estrategia que implementó D3 fue "involucrar a los alumnos, comprometiéndolos a participar y tomando lista en diferentes momentos". Para D2 fue muy importante "ser tolerante" e implementó una estrategia alternativa de envíos de tareas "por chat o por e-mail" cuando había problemas con la plataforma. También "estableció reglas con los alumnos” y configuró las sesiones virtuales de clase "para que los alumnos no pudieran sacar a los compañeros ni estuvieran jugando". Por su parte, D5 les mostró "fotos o videos (...) sobre piezas de hardware y procedimientos con ellos". D1 utilizó "software simulador” y "preparó algunos nuevos materiales de aprendizaje".

\section{¿Cómo se sentían los docentes con el reto de impartir materias prácticas en línea?}

La incertidumbre fue el sentimiento común entre los docentes. Para D4 "al principio sí fue un reto por la preparación del material, que estaba listo para exponerse de manera presencial (...). Me sentí un poco perdido por no saber cómo iban avanzando mis alumnos (...). Luego me sentí muy a gusto. Lo vi como una oportunidad de hacer una práctica real de lo que había aprendido de Teams". D5 coincidió: “Al principio, no conocer la plataforma (me hizo sentir) incierto, con desconocimiento. Luego contento de vivir la experiencia". Para D3 también "fue un reto, una incertidumbre por la situación, por los alumnos". D2 se "sentía 


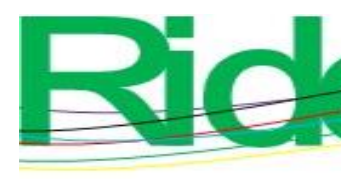

Revista Iberoamericana para la
Investigación y el Desarrollo Educativo
ISSN $2007-7467$

Fuente: Elaboración propia

Los alumnos coincidieron con lo expuesto por los docentes. "Primero nos explicaban con diapositivas o documentos, luego nos ponían a hacer prácticas y ejercicios a realizar y después mandarlos para calificarlos" (E1). "Mediante la pantalla compartida podíamos seguir los ejercicios que el profesor nos ensañaba" (E3). "Se explicaba la clase y nos asignaban diferentes ejercicios que teníamos que desarrollar" (E5).

\section{¿Cómo fue la evaluación del curso?}

Para la dinámica de la evaluación, D2 aplicó exámenes teóricos de opción múltiple y exámenes prácticos. Ambos se tenían que contestar durante la sesión de clase y los alumnos tenían un tiempo reducido para responderlos. Para D3, las evaluaciones fueron prácticas y consistieron en la elaboración de un programa en una hora como máximo, dentro de la sesión de clase. D4 también hizo exámenes teóricos, pero, por el contrario, los alumnos los contestaron fuera del horario de clase. D4 rediseñó sus exámenes para que las preguntas no fueran tan obvias en su respuesta, sino que mostraran casos aplicados que requirieran cierto razonamiento. D5 evaluó el curso solamente con las actividades realizadas a lo largo del curso. Decidió esto porque antes de que la contingencia iniciara, el grupo ya había resuelto un examen.

Los alumnos, por su parte, expresaron que la evaluación de sus materias prácticas fue buena y que no varió en comparación con la modalidad presencial. "Prácticamente (fue) en la misma forma que en la modalidad presencial" (E1). "Fue una buena evaluación, ya que se tomó en cuenta tanto los trabajos en clase, las tareas y, por supuesto, los exámenes” (E2). "Me fue bastante bien. No variaba mucho la manera de evaluar a la presencial" (E6).

\section{Alumnos \\ ¿Cómo fue la comunicación maestro-alumno?}

Los cinco profesores entrevistados coincidieron en que la comunicación a través de Teams fue buena, "bastante fluida" (D1), "muy abierta, yo siempre estaba disponible para ellos (...), insistí mucho en que se comunicaran" (D3), "muy fluida, (los alumnos fueron) explícitos en sus mensajes, su confianza fue aumentando y no tuvieron límite” (D4), “excelente y con la confianza de decir si tienen dudas o necesidades" (D5). D2 profundizó: 


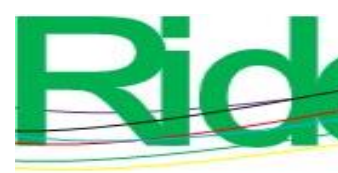

Revista Iberoamericana para la Investigación y el Desarrollo Educativo ISSN $2007-7467$

“(Los alumnos) me mandaban muchos mensajes y los contestaba en ese mismo rato, incluso hasta muy noche".

Los alumnos también coincidieron en que la comunicación con sus profesores fue buena: "(Los profesores) estaban al pendiente de que pudiéramos entender las cosas" (E2). “(La comunicación estuvo) bien. Es algo más personal y confidente, así que me parece estupenda” (E3). “(La comunicación estuvo) bien, normal, más personal en comparación a cuando es presencial" (E4).

\section{¿Cómo se resolvían las dudas de los alumnos?}

Durante las sesiones de clase había retroalimentación sobre las prácticas (D1), pero también solución de dudas (D3, D4), las cuales se podían manifestar por chat o a través del micrófono (D4, D5). Otro medio de solución de dudas era a través de la funcionalidad de “compartir pantalla", "ellos compartían su escritorio y se abría debate a toda la clase" (D3), “(yo) compartía mi pantalla y hacía el procedimiento paso a paso (...); si no captaban, ellos compartían su pantalla y yo les iba diciendo el procedimiento paso a paso" (D2). Las dudas podían surgir en clase o fuera de ella. Sin embargo, se manifestaron y se solucionaron principalmente a través de la herramienta Teams. "En Base de datos avanzada hacían muchas preguntas (...). Hay sesiones grabadas en donde casi todo el tiempo son preguntas (...). En el caso de programación casi no preguntaban durante clase y luego me enviaban todas las dudas por chat, excepcionalmente por Whatsapp" (D4). "Los alumnos no usaron el correo electrónico. Prefirieron el chat. La solución de dudas fuera de clase fue a través del chat" (D1). Además de las preguntas aisladas, D2 y D4 impartieron sesiones de asesoría más extensas. "A una alumna le di asesoría personalizada y le dediqué mucho tiempo" (D4). "Impartí dos sesiones virtuales para asesoría especial a través de Teams” (D2).

Los alumnos destacaron que el chat de Teams fue de mucha utilidad para manifestar y aclarar sus dudas (E3, E4, E5, E6). Además, no todo se lo preguntaban al profesor: "No acudía tanto a ellos (a los profesores). Prefería buscar información por mis medios, en internet, para resolver mis preguntas" (E2). 


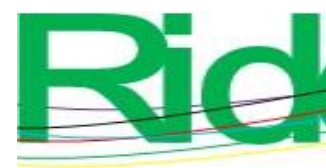

Revista Iberoamericana para la Investigación y el Desarrollo Educativo ISSN $2007-7467$

Tabla 6. Ventajas de la modalidad en línea que fueron percibidas por los docentes

\begin{tabular}{|l|c|c|c|c|c|}
\hline Ventajas percibidas & D1 & D2 & D3 & D4 & D5 \\
\hline Me resulta más práctico / cómodo & $\checkmark$ & & & $\checkmark$ & \\
\hline No hay necesidad de trasladarse físicamente al campus & $\checkmark$ & & & $\checkmark$ & \\
\hline $\begin{array}{l}\text { Es posible estar más al pendiente de la comunicación con los } \\
\text { alumnos }\end{array}$ & & $\checkmark$ & & & $\checkmark$ \\
\hline Sin tantos distractores como en las sesiones presenciales & & $\checkmark$ & $\checkmark$ & $\checkmark$ & \\
\hline Se ahorra tiempo & & & $\checkmark$ & & \\
\hline Es posible usar más herramientas tecnológicas & & & $\checkmark$ & & $\checkmark$ \\
\hline $\begin{array}{l}\text { Es más conveniente para complementar la docencia con otras } \\
\text { actividades personales }\end{array}$ & & & & $\checkmark$ & \\
\hline Es posible grabar las sesiones y verlas posteriormente & & & & $\checkmark$ & \\
\hline Se comparte contenido digital con mayor facilidad & & & & $\checkmark$ & $\checkmark$ \\
\hline
\end{tabular}

Fuente: Elaboración propia

\section{¿Qué tan satisfechos están los alumnos con la modalidad en línea?}

Los estudiantes fueron muy mesurados en sus respuestas y mostraron un nivel promedio de satisfacción. Ninguno se mostró altamente satisfecho o insatisfecho. Algunos comentarios fueron: "La impartición de clase fue adecuada" (E2). "En cuanto a las clases no tengo queja alguna. Solamente un poco insatisfecha por los problemas propios de la aplicación” (E4). "Lo normal. Fue una buena experiencia” (E3). "Diría que normal. No fue algo espectacular ni decepcionante" (E5). "Satisfecha. Lo justo. Fue una experiencia nueva, pero hasta ahí" (E6).

\section{¿Qué desventajas se perciben sobre la docencia de materias prácticas en línea?}

La respuesta común de los docentes fue que la principal desventaja es que se requiere tener acceso a internet y por lo menos un equipo para conectarse. Se reflexionó acerca de que algunos estudiantes no tienen recursos económicos para adquirir los equipos necesarios (D3) y sobre la incapacidad de los proveedores locales de internet para ofrecer acceso continuo de alta velocidad en las diferentes zonas geográficas, especialmente cuando hay muchos usuarios conectados (D3, D4). También, dos entrevistados comentaron concretamente sobre las desventajas de haber tenido que impartir clases en línea sin haberlo anticipado con suficiente antelación. Al respecto, D4 subrayó la apatía que puede surgir en los alumnos por tomar las clases en línea cuando originalmente estaban previstas para ser presenciales. D5 puso en relieve que el sorpresivo inicio de las clases en línea impidió que los docentes 


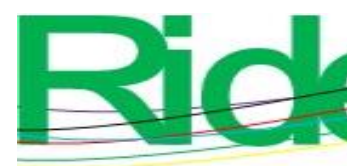

Revista Iberoamericana para la
Investigación y el Desarrollo Educativo
ISSN $2007-7467$

contaran con una preparación previa tanto en su formación como en el uso de las herramientas tecnológicas de aprendizaje.

Algunos alumnos coincidieron sobre los aspectos técnicos: "La mayor desventaja fue con el internet, ya que me quedaba varios días sin conexión y no podía entrar a las clases, entonces perdía exposiciones o me atrasaba en los trabajos y a veces me costaba entender" (E6). "A veces fallaba el audio y como no podía escuchar bien, me llegué a quedar dormida" (E4). Otros comentaron concretamente sobre la atención: "No todos (los alumnos) le ponían atención (a la clase) o de plano no aprendían" (E1). "La fuerza de voluntad se requiere mucho más, ya que al estar en casa uno se distrae prácticamente con cualquier cosa y deja de prestar atención a lo que está impartiendo el profesor” (E2).

\section{¿Estarían dispuestos los docentes a seguir enseñando en línea y los alumnos a seguir cursando sus materias en línea cuando la pandemia haya terminado?}

Los cinco profesores entrevistados dijeron que estarían dispuestos a impartir materias prácticas en línea, incluso si ya no hubiera contingencia, teniendo contenidos preparados (D1, D4, D5) y materiales en video (D5), así como tiempo para prepararlos (D1, D4). D3 dijo que esta modalidad "ayudaría a administrar los grupos grandes en la universidad". Para D2 “podría ser complejo acoplar los horarios de materias virtuales y presenciales (...) si el formato de las materias virtuales implicara conectarse en vivo a ciertas horas (como en esta ocasión)”. Hizo referencia a esto porque en el campus físico de la universidad una buena conexión a internet y un espacio con privacidad son recursos que no se consiguen fácilmente a cualquier hora.

El caso de los alumnos fue distinto. La mayoría de los entrevistados optaría por la modalidad presencial (E4, E6). "Si bien la herramienta de Microsoft Teams ofrece muy buenas herramientas tanto para alumnos como maestros, sinceramente si fuese por mi decisión, prefiero seguir con el formato presencial" (E1). "Sigo a favor de la modalidad presencial” (E5). E2 comentó: "Sí tomaría mis clases en línea, aunque no es un secreto decir que si la plataforma corrigiera sus errores sería mucho más confortable” (E2) y para E3 “es indiferente llevarlas de un modo u otro". 


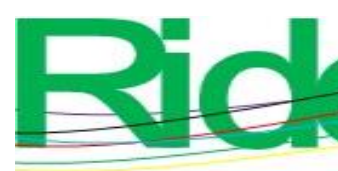

Revista Iberoamericana para la
Investigación y el Desarrollo Educativo
ISSN $2007-7467$

necesarios para contestar a través del chat, incluso desde el teléfono celular, lo cual fue percibido como muy conveniente. Durante las sesiones de clase las dudas también se podían resolver de viva voz, o a través del micrófono. Por otra parte, si bien es cierto que algunos alumnos enfrentaron retos familiares y de trabajo, fueron más quienes en algún momento tuvieron problemas relacionados con la disponibilidad de recursos tecnológicos tales como los equipos de cómputo o la conexión a internet.

Los docentes se mostraron contentos con la experiencia de haber impartido clases en línea. Encontraron varias ventajas de trabajar de esta manera. Por ejemplo: al no estar presencialmente en el campus universitario, no hay tantas interrupciones o suspensiones de clase por otros eventos, es más cómodo, sin traslados físicos, la comunicación con los alumnos es mejor, por la naturaleza de esta modalidad, se aprovecha más la tecnología, y es más fácil compartir recursos digitales con los estudiantes. Sin embargo, todos coincidieron en que la principal desventaja es la necesidad de contar con recursos tecnológicos disponibles. También todos dijeron que aceptarían impartir materias en línea aun cuando la contingencia se hubiera terminado. En este sentido, los docentes manifestaron que les gustaría mejorar el formato de presentación de sus clases, agregando mayor interactividad, grabando videos de buena calidad y bien planificados. También dijeron que las evaluaciones podrían mejorarse incorporando otros métodos o técnicas. Asimismo, expresaron que sería apropiado realizar mayor planeación y dosificación de contenidos.

Los alumnos, por su parte, percibieron muy positivamente la comunicación con sus maestros y la evaluación de sus materias. Reconocieron que principalmente enfrentaron problemas técnicos y aunque estuvieron satisfechos con la experiencia en línea, prefieren la modalidad presencial. Los estudiantes también aceptaron que en su casa hay distractores que pueden provocar que se desconcentren y no pongan la debida atención a la clase. En cuanto a la dinámica de las sesiones, manifestaron que les gustaría que se incluyeran mayor número de actividades interactivas.

La figura 1 muestra los hechos más importantes que reportaron los docentes, y la figura 2 los aspectos más importantes expresados por los alumnos. 
Figura 1. Resumen de las respuestas de los docentes
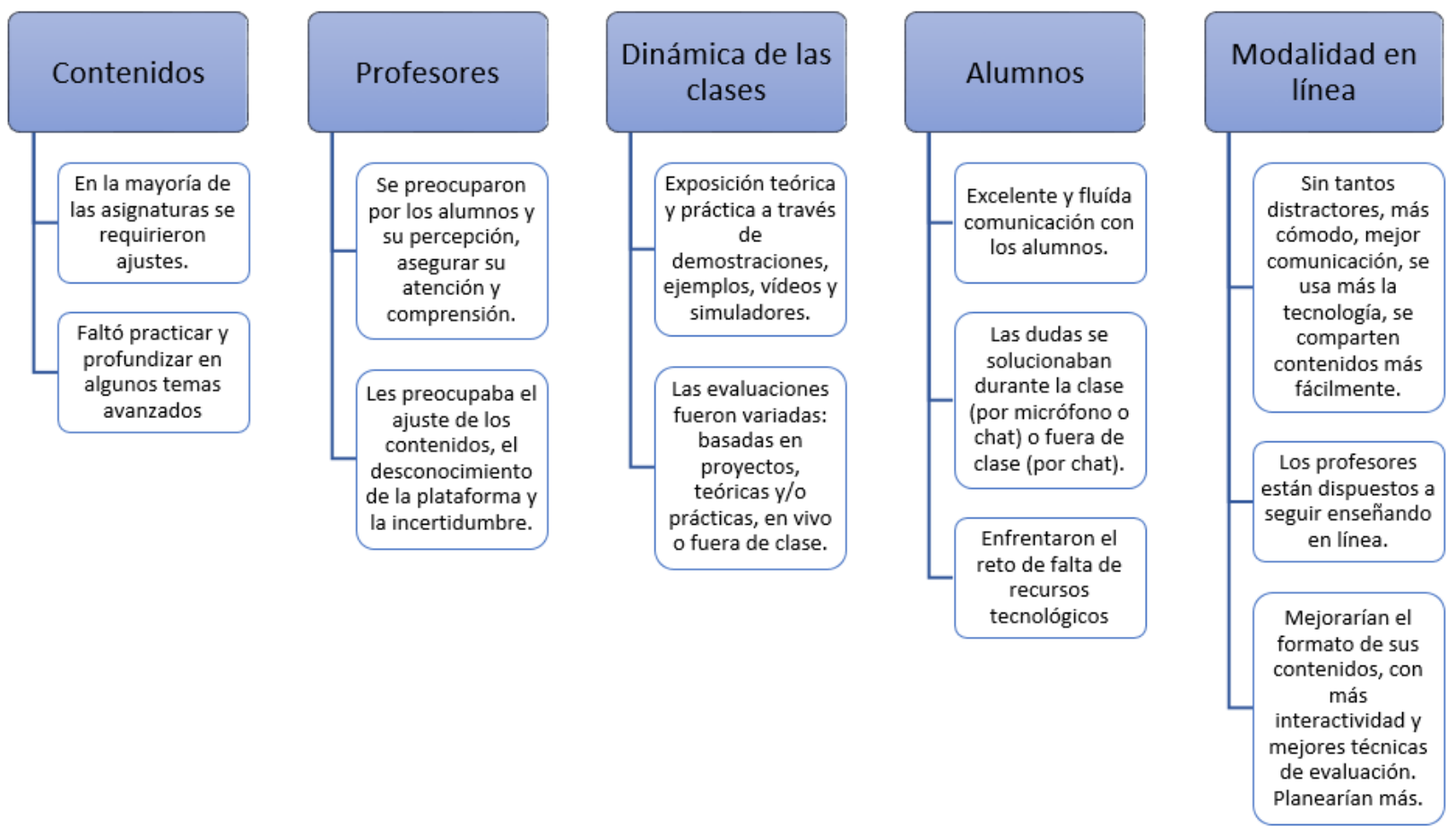

Fuente: Elaboración propia

Figura 2. Resumen de las respuestas de los alumnos
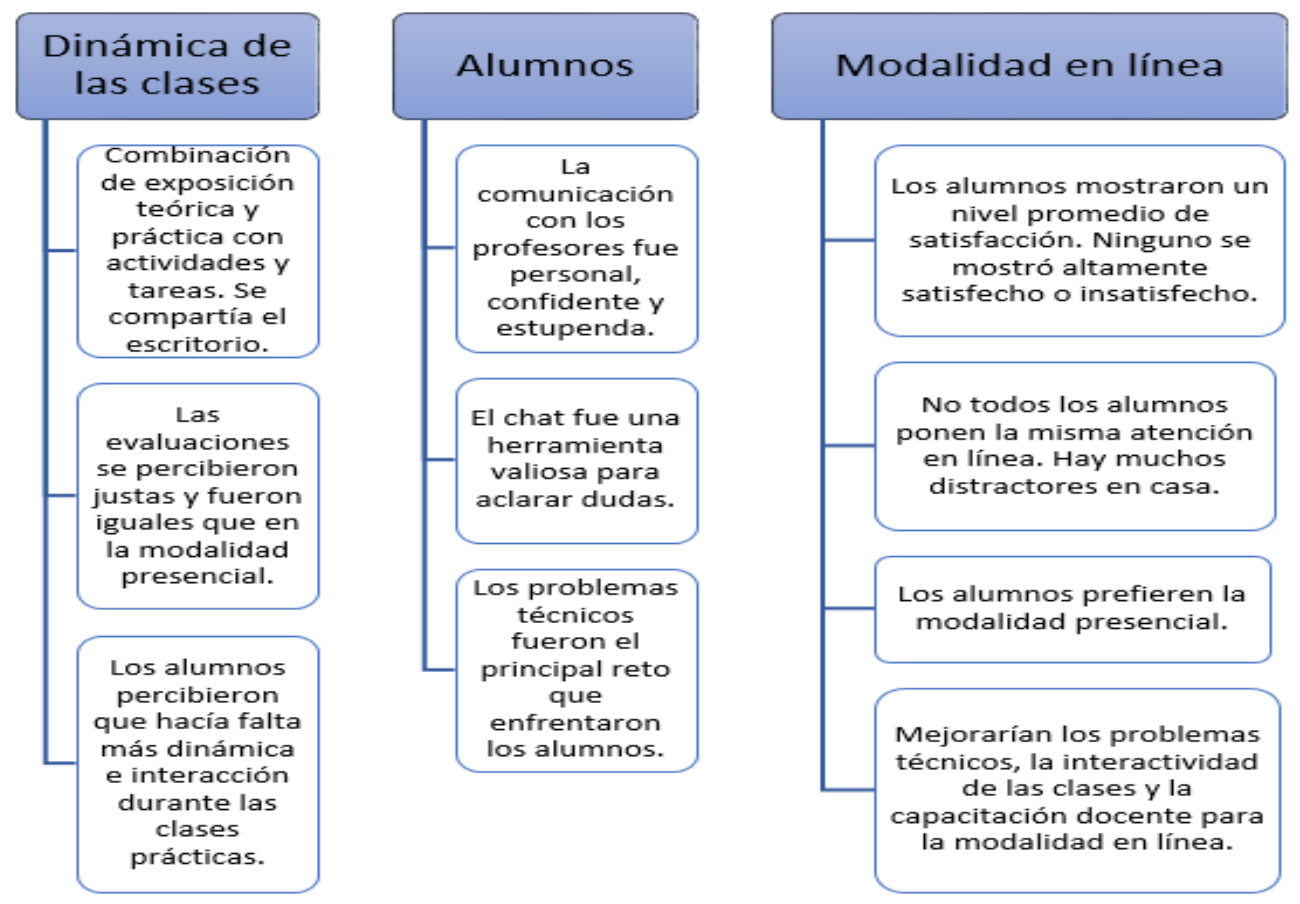

Fuente: Elaboración propia 


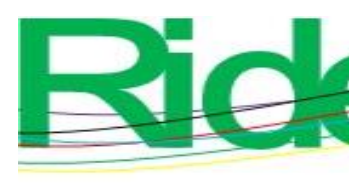

Revista Iberoamericana para la Investigación y el Desarrollo Educativo ISSN $2007-7467$

\section{Limitaciones y delimitaciones}

Esta investigación estuvo delimitada a cinco docentes de la UNX que imparten asignaturas prácticas en el programa educativo de licenciatura en Tecnologías de la Información y a seis alumnos de estos mismos cursos. Las preguntas estuvieron orientadas únicamente al tiempo de contingencia sanitaria en el que tuvieron que impartir sus asignaturas en línea. Es decir, desde el 17 de marzo hasta el 17 de mayo de 2020.

\section{Contraste de los hallazgos con la literatura analizada}

Nuestros hallazgos coinciden con Sinha et al. (2020) en relación con la utilidad de la plataforma Microsoft Teams, la cual fue bien valorada por maestros y alumnos. Por otra parte, los retos mencionados por los docentes y alumnos son similares con las problemáticas expuestas por Feng et al. (2020) y por Ping et al. (2020). También los enfoques de enseñanza que adoptaron los profesores coinciden con el modelo de aprendizaje basado en problemas propuesto por estos últimos autores. Finalmente, encontramos que las interrogantes planteadas por Cerf (2020) tienen fundamento. Al respecto, nuestros resultados sugieren que en una futura etapa postcovid-19, la educación en línea sería la preferida por los profesores.

\section{Conclusiones}

En esta investigación se encontró que, en las clases universitarias en modalidad virtual, Microsoft Teams realmente hace posible la docencia totalmente conectada, la comunicación efectiva con los estudiantes, así como la recepción y evaluación de tareas. Asimismo, facilita el compartir contenidos. Sin embargo, representa solo un medio a través del cual se desarrollan los procesos de enseñanza-aprendizaje. El profesor tiene la responsabilidad de diseñar, subir, organizar y explicar los materiales de aprendizaje.

Además, en las asignaturas prácticas docentes y alumnos enfrentan el reto de cubrir temas que presencialmente abordarían en un laboratorio. Para lograr este objetivo, los docentes utilizan demostraciones en vivo, videotutoriales, simuladores e incluso explicaciones teóricas sobre procedimientos prácticos. En la presente investigación se encontró que Microsoft Teams es una herramienta muy útil en este sentido, pues permite compartir la pantalla, videograbar las sesiones y responder dudas a través de audio o de chat. Sin embargo, no sustituye a la buena organización docente ni a un buen diseño del curso. Los docentes reconocieron que, a pesar de que la mayor parte de los contenidos se pudo cubrir 


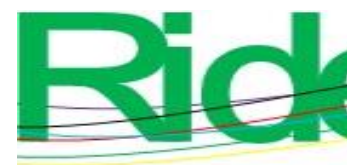

Revista Iberoamericana para la
Investigación y el Desarrollo Educativo
ISSN $2007-7467$

queda por estudiar con el objetivo de caracterizar su adopción y apropiación, así como las percepciones, tendencias y cambios institucionales derivados de la virtualidad obligatoria a causa de la COVID-19.

\section{Agradecimientos}

Los autores agradecen a la Universidad Autónoma de Tamaulipas, México, pues el proyecto (32-UATINVES20) al que pertenece esta publicación recibió financiamiento interno al resultar seleccionado en la convocatoria de investigación UAT 2020-2.

\section{Referencias}

Belvin, P. (2018). Revitalizing student worker training: Using office 365 to train and manage your student workforce. Proceedings ACM SIGUCCS User Services Conference, 169-172. Doi: https://doi.org/10.1145/3235715.3235734

Cerf, V. G. (2020). Implications of the COVID-19 pandemic. Communications of the ACM, 63(6), 7. Doi: https://doi.org/10.1145/3397262

Feng, X.-L., Yu, T., Hu, X.-C. and Fan, K.-Y. (2020). Brief discussion about the impact of the Coronavirus Disease 2019 on Teaching in Colleges and Universities of China. 2020 International Conference on E-Commerce and Internet Technology (ECIT), 167-170. Doi: https://doi.org/10.1109/ECIT50008.2020.00044

Ferrario, L., Maffioli, A., Bondurri, A. A., Guerci, C., Lazzarin, F. and Danelli, P. (2020). COVID-19 and surgical training in Italy: Residents and young consultants perspectives from the battlefield. American Journal of Surgery, (xxxx). Doi: https://doi.org/10.1016/j.amjsurg.2020.05.036

Ghosh, A., Gupta, R. and Misra, A. (2020). Telemedicine for diabetes care in India during COVID19 pandemic and national lockdown period: Guidelines for physicians. Diabetes and Metabolic Syndrome: Clinical Research and Reviews, 14(4), 273-276. Doi: https://doi.org/10.1016/j.dsx.2020.04.001

Haleem, A., Javaid, M., Vaishya, R. and Deshmukh, S. G. (2020). Areas of academic research with the impact of COVID-19. American Journal of Emergency Medicine, (xxxx), 5-7. https://doi.org/10.1016/j.ajem.2020.04.022

Halstead-Nussloch, R., Dickson, C., Greer, K., Siddiqui, S. and Tumuluri, R. D. (2019). Recursive online class: Advanced web application class prototypes online educational 


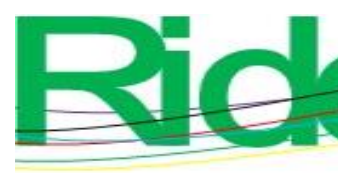

Revista Iberoamericana para la
Investigación y el Desarrollo Educativo
ISSN $2007-7467$

resources. ACMSE 2019 - Proceedings of the 2019 ACM Southeast Conference, 275276. Doi: https://doi.org/10.1145/3299815.3314475

Hodgson, J. C. and Hagan, P. (2020). Medical Education Adaptations During a Pandemic: Transitioning to Virtual Student Support. Medical Education, 44(0). Doi: https://doi.org/10.1111/medu.14177

Owens, K., Weismann, C. and Suskin, M. (2019). Bringing campus experience to online classes. Proceedings ACM SIGUCCS User Services Conference, 61-63. Doi: https://doi.org/10.1145/3347709.3347831

Panesar, K., Dodson, T., Lynch, J., Bryson-Cahn, C., Chew, L. and Dillon, J. (2020). Evolution of COVID-19 Guidelines for University of Washington Oral and Maxillofacial Surgery Patient Care. Journal of Oral and Maxillofacial Surgery. Doi: https://doi.org/https://doi.org/10.1016/j.joms.2020.04.034

Pather, N., Blyth, P., Chapman, J. A., Dayal, M. R., Flack, N. A. M. S., Fogg, Q. A., ... Lazarus, M. D. (2020). Forced Disruption of Anatomy Education in Australia and New Zealand: An Acute Response to the Covid-19 Pandemic. In Anatomical Sciences Education. Doi: https://doi.org/10.1002/ase.1968

Ping, Z., Fudong, L. and Zheng, S. (2020). Thinking and Practice of Online Teaching under COVID-19 Epidemic. 2020 IEEE 2nd International Conference on Computer Science and Educational Informatization (CSEI), 165-167.

Price, A. T., Henke, L. E., Maraghechi, B., Kim, T., Spraker, M. B., Hugo, G. D., ... Knutson, N. C. (2020). Implementation of a Novel Remote Physician SBRT Coverage Process during the Coronavirus Pandemic. MedRxiv, Doi: https://doi.org/10.1101/2020.04.09.20059857

Sinha, S. S., Sharma, G. and Cullen, M. W. (2020). The Crucible of Crisis. Journal of the American College of Cardiology, (April), 5-7. Doi: https://doi.org/10.1016/j.jacc.2020.04.012

Tofade, T. and Daftary, M. N. (2020). Managing college operations during the coronavirus outbreak. Journal of the American Pharmacists Association, 1-2. Doi: https://doi.org/10.1016/j.japh.2020.03.027 


\begin{tabular}{|c|c|}
\hline Rol de Contribución & Autor (es) \\
\hline Conceptualización & Ramón Ventura Roque Hernández \\
\hline Metodología & $\begin{array}{l}\text { Ramón Ventura Roque Hernández, José Balderas Solís, Carlos } \\
\text { Manuel Juárez Ibarra } \\
\text { Grado de contribución: igual }\end{array}$ \\
\hline Software & N/A \\
\hline Validación & $\begin{array}{l}\text { Ramón Ventura Roque Hernández, Rolando Salazar Hernández } \\
\text { Grado de contribución: igual }\end{array}$ \\
\hline Análisis Formal & $\begin{array}{l}\text { Ramón Ventura Roque Hernández, José Balderas Solís, } \\
\text { Rolando Salazar Hernández, Adán López Mendoza } \\
\text { Grado de contribución: igual }\end{array}$ \\
\hline Investigación & $\begin{array}{l}\text { José Balderas Solís, Ramón Ventura Roque Hernández } \\
\text { Grado de contribución: igual }\end{array}$ \\
\hline Recursos & $\begin{array}{l}\text { José Balderas Solís, Ramón Ventura Roque Hernández } \\
\text { Grado de contribución: igual }\end{array}$ \\
\hline Curación de datos & $\begin{array}{l}\text { Carlos Manuel Juárez Ibarra, Adán López Mendoza } \\
\text { Grado de contribución: igual }\end{array}$ \\
\hline $\begin{array}{l}\text { Escritura - Preparación del } \\
\text { borrador original }\end{array}$ & $\begin{array}{l}\text { Ramón Ventura Roque Hernández (Principal), } \\
\text { José Balderas Solís (Apoyo) }\end{array}$ \\
\hline $\begin{array}{l}\text { Escritura - Revisión y } \\
\text { edición }\end{array}$ & $\begin{array}{l}\text { José Balderas Solís (Principal), } \\
\text { Ramón Ventura Roque Hernández (Principal), } \\
\text { Carlos Manuel Juárez Ibarra (Apoyo) }\end{array}$ \\
\hline Visualización & $\begin{array}{l}\text { Rolando Salazar Hernández, Adán López Mendoza } \\
\text { Grado de contribución: igual }\end{array}$ \\
\hline Supervisión & Ramón Ventura Roque Hernández \\
\hline $\begin{array}{l}\text { Administración de } \\
\text { Proyectos }\end{array}$ & Ramón Ventura Roque Hernández \\
\hline Adquisición de fondos & Ramón Ventura Roque Hernández \\
\hline
\end{tabular}

